Supporting Information

\title{
Atomic-Scale Tailoring Organic Cation of Layered Ruddlesden-Popper Perovskite Compounds
}

Han Pan ${ }^{a}$, Xiaojuan Zhao ${ }^{a}$, Xiu Gong ${ }^{a}$, Yan Shen ${ }^{a}$, and Mingkui Wang ${ }^{a}$ *

${ }^{a}$ Wuhan National Laboratory for Optoelectronics, School of Optical and Electronic Information, Huazhong University of Science and Technology, Wuhan 430074, Hubei, P. R. China 


\section{Experimental Section}

$\mathrm{PbO}$ (99.9\%), hydroiodic acid (57 wt.\% in $\mathrm{H}_{2} \mathrm{O}$, distilled, stabilized, 99.95\%), and hypophosphorous acid solution ( $50 \mathrm{wt} . \%$ in $\mathrm{H}_{2} \mathrm{O}$ ) were purchased from Sigma-Aldrich and used as received. Phenethylamine, 4-fluorophenethylamine, 2-(4-fhlorophenyl) ethylamine, and 2-(4-bromophenyl)ethylamine were purchased from Innochem Technology Corp. and used as received.

Synthesis of Aromatic Ammonium Iodide. H-PEAI, F-PEAI, Cl-PEAI, and Br-PEAI: hydroiodic acid (12.3 g, $0.055 \mathrm{~mol}$.), respectively, reacted with phenethylamine (6.1g, $0.05 \mathrm{~mol}$.), 4-Fluorophenethylamine (7.0 g, $0.05 \mathrm{~mol}$.), 2-(4-Chlorophenyl) ethylamine (7.8 g, $0.05 \mathrm{~mol}$.), and 2-(4-Bromophenyl)ethylamine (10.1 g, $0.05 \mathrm{~mol}$.) for 2 hours under ice bath, and the other preparation steps adopted the same method above. The composition and structure of aromatic ammonium iodide were confirmed by ${ }^{1} \mathrm{H}$ NMR (Figure S1).

Synthesis of F-PEA ${ }_{2} \mathrm{PbI}_{4}$. $\mathrm{PbO}(0.25 \mathrm{mmol}, 56 \mathrm{mg})$ powder was dissolved in a mixture of $5 \mathrm{~mL}$ of hydroiodic acid and $1 \mathrm{~mL}$ of hypophosphorous acid solution by heating to boiling under stirring. After powders completely dissolved, solid F-PEAI $(0.5 \mathrm{mmol}, 133.5 \mathrm{mg})$ was added into the previous solution, causing the orange precipitation, which could be re-dissolve after continuous stirring. During cooling the solution to room temperature, orange plate-like crystals precipitated out. The crystals were obtained by suction filtration and vacuum drying. Yield: $228 \mathrm{mg}(91.6 \%$ based on total $\mathrm{Pb}$ content) (Table $\mathrm{S} 1)$.

Synthesis of Cl-PEA ${ }_{2} \mathrm{PbI}_{4}$. For the synthesis of $\mathrm{Cl}-\mathrm{PEA}_{2} \mathrm{PbI}_{4}$, similar synthetic procedures were followed. Orange plate-like crystals were obtained. Yield: $236 \mathrm{mg}$ (92.1\% based on total $\mathrm{Pb}$ content).

Synthesis of $\mathrm{Br}-\mathrm{PEA}_{2} \mathrm{PbI}_{4}$. For the synthesis of $\mathrm{Br}-\mathrm{PEA}_{2} \mathrm{PbI}_{4}$, similar synthetic procedures were followed. Orange plate-like crystals were obtained. Yield: $252 \mathrm{mg}$ (90.3\% based on total $\mathrm{Pb}$ content).

Solution preparation. The perovskite solution $(0.2 \mathrm{M})$ of $\mathrm{H}-\mathrm{PEA}_{2} \mathrm{MA}_{2} \mathrm{~Pb}_{3} \mathrm{I}_{10}$ was comprised of H-PEAI, MAI and $\mathrm{PbI}_{2}$ with 2:2:3 molar ratio in the anhydrous DMF 
solvent. And the preparation of X-PEA $\mathrm{MA}_{2} \mathrm{~Pb}_{3} \mathrm{I}_{10}(\mathrm{~F}, \mathrm{Cl}, \mathrm{Br})$ perovskite solution was followed. The solutions were stirred at $70{ }^{\circ} \mathrm{C}$ for $2 \mathrm{~h}$ under inert atmosphere.

Device fabrication

The FTO glass was sequentially cleaned with detergent, deionized water, acetone and ethanol, and then treated in UV-ozone for $30 \mathrm{~min}$. A $50 \mathrm{~nm}$ thick $\mathrm{NiO}_{\mathrm{x}}$ was spincoated onto the FTO substrate at $2000 \mathrm{rpm}$ for $30 \mathrm{~s}$ and annealed at $100{ }^{\circ} \mathrm{C}$ for $10 \mathrm{~min}$, $480{ }^{\circ} \mathrm{C}$ for $20 \mathrm{~min}$ in air. After $\mathrm{HPEA}_{2} \mathrm{MA}_{2} \mathrm{~Pb}_{3} \mathrm{I}_{10}$ and $\mathrm{X}-\mathrm{PEA}_{2} \mathrm{MA}_{2} \mathrm{~Pb}_{3} \mathrm{I}_{10}(\mathrm{~F}, \mathrm{Cl}, \mathrm{Br})$ films were spin-coated at $3000 \mathrm{rpm}$ for $45 \mathrm{~s}, \mathrm{PC}_{61} \mathrm{BM}\left(20 \mathrm{mg} \cdot \mathrm{mL}^{-1}\right.$ in chlorobenzene) and $\mathrm{BCP}\left(0.5 \mathrm{mg} \cdot \mathrm{mL}^{-1}\right.$ in isopropanol) were sequentially coated at $2000 \mathrm{rpm}$ for $40 \mathrm{~s}$ and $4000 \mathrm{rpm}$ for $40 \mathrm{~s}$, respectively. Finally, $100 \mathrm{~nm} \mathrm{Ag}$ was deposited by thermal evaporation.

\section{Characterization}

XRD measurements were performed with a Shimadzu XRD-6100 diffractometer with $\mathrm{Cu} \mathrm{K} \alpha$ radiation. Single-crystal X-ray diffraction data were collected using 39 $\mathrm{XtaLAB}$ PRO MM007HF $\mathrm{Cu}$ with $\mathrm{Cu} \mathrm{K} \alpha$ radiation. All structures were solved by the APEX3 software. The surface morphology of films and energy dispersive spectrometer images were characterized with FEI Nova Nano SEM 450. Visible absorption spectra were recorded using a PerkinElmer Lambda1050 spectrophotometer. The steady-state photoluminescence (PL) spectrum was measured using Horiba Jobin Yvon system with an excitation laser beam at $532 \mathrm{~nm}$ and a repetition rate of $76 \mathrm{MHz}$. The time-resolved luminescence decays were obtained with time-correlated single photo counting system (PicoHarp 300, PicoQuant GmbH). The excitation light source was Ti:Sapphire laser (Mira 900, Coherent; 76 MHz, 130 fs). UPS and XPS measurements were performed by a Kratos AXIS Ultra-DLD ultra-high-vacuum photoemission spectroscopy system with an $\mathrm{Al} \mathrm{K} \alpha$ radiation source. The film thickness was measured with a profile-meter (Veeco Dektak 150). The ${ }^{1} \mathrm{H}$ NMR spectra was measured on a Bruker-AF301 AT 400 $\mathrm{MHz}$ spectrometer.

The photocurrent-voltage $(\mathrm{J}-\mathrm{V})$ characteristics of $\mathrm{XPEA}_{2} \mathrm{MA}_{2} \mathrm{~Pb}_{3} \mathrm{I}_{10}(\mathrm{X}=\mathrm{H}, \mathrm{F}, \mathrm{Cl}$, $\mathrm{Br}$ ) solar cells were obtained by a Keithley model 2400 digital source meter. A xenon 
light source solar simulator (450 W, Oriel, model 9119) with AM 1.5G filter (Oriel, model 91192) was used to give an irradiance of $100 \mathrm{~mW} \mathrm{~cm}^{-2}$ at the surface of the solar cells. The incident photon conversion efficiency (IPCE) measurement was obtained with alternating current $(\mathrm{AC})$ model $(130 \mathrm{~Hz})$.

(a)

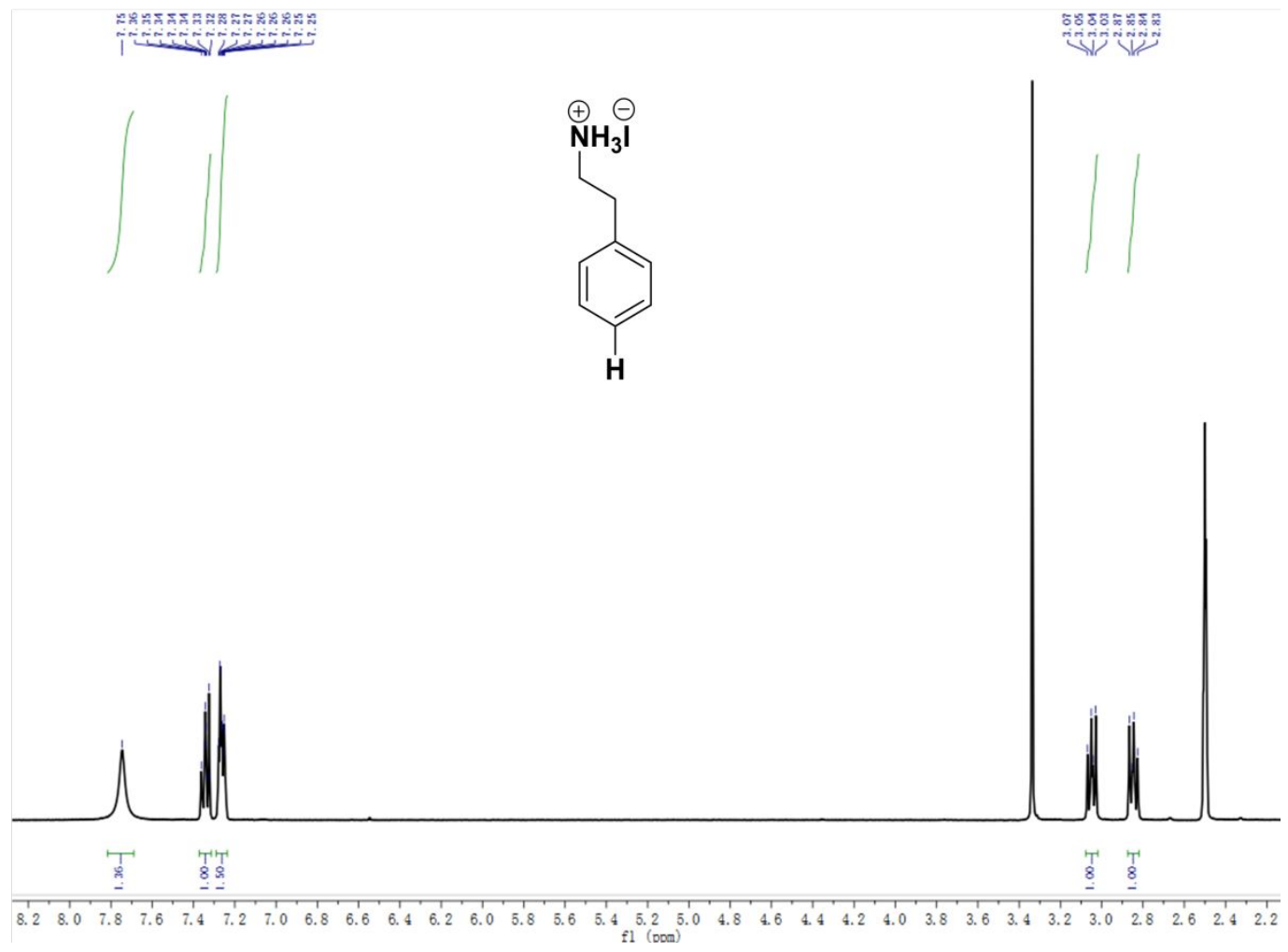


(b)

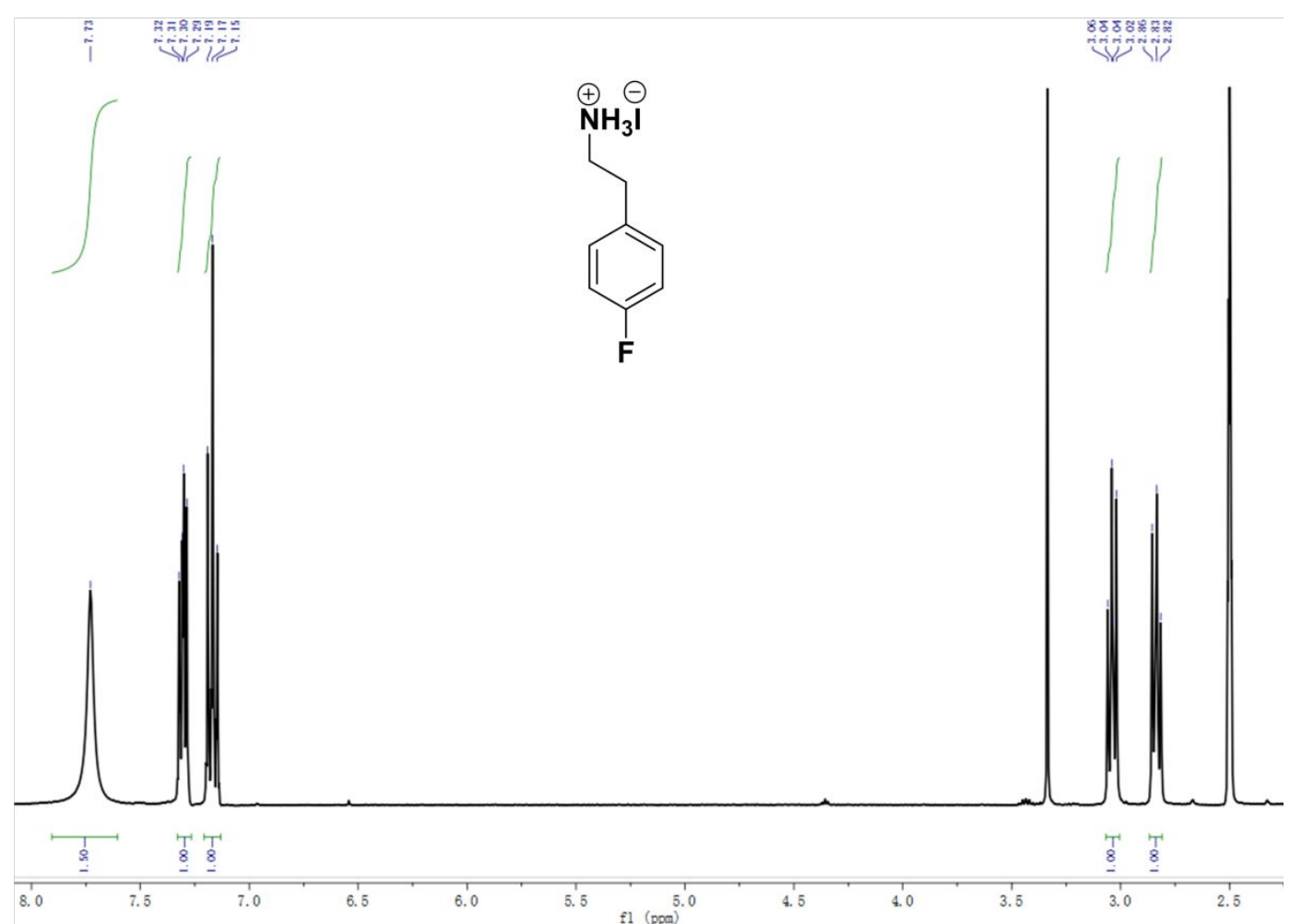

(c) i

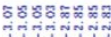

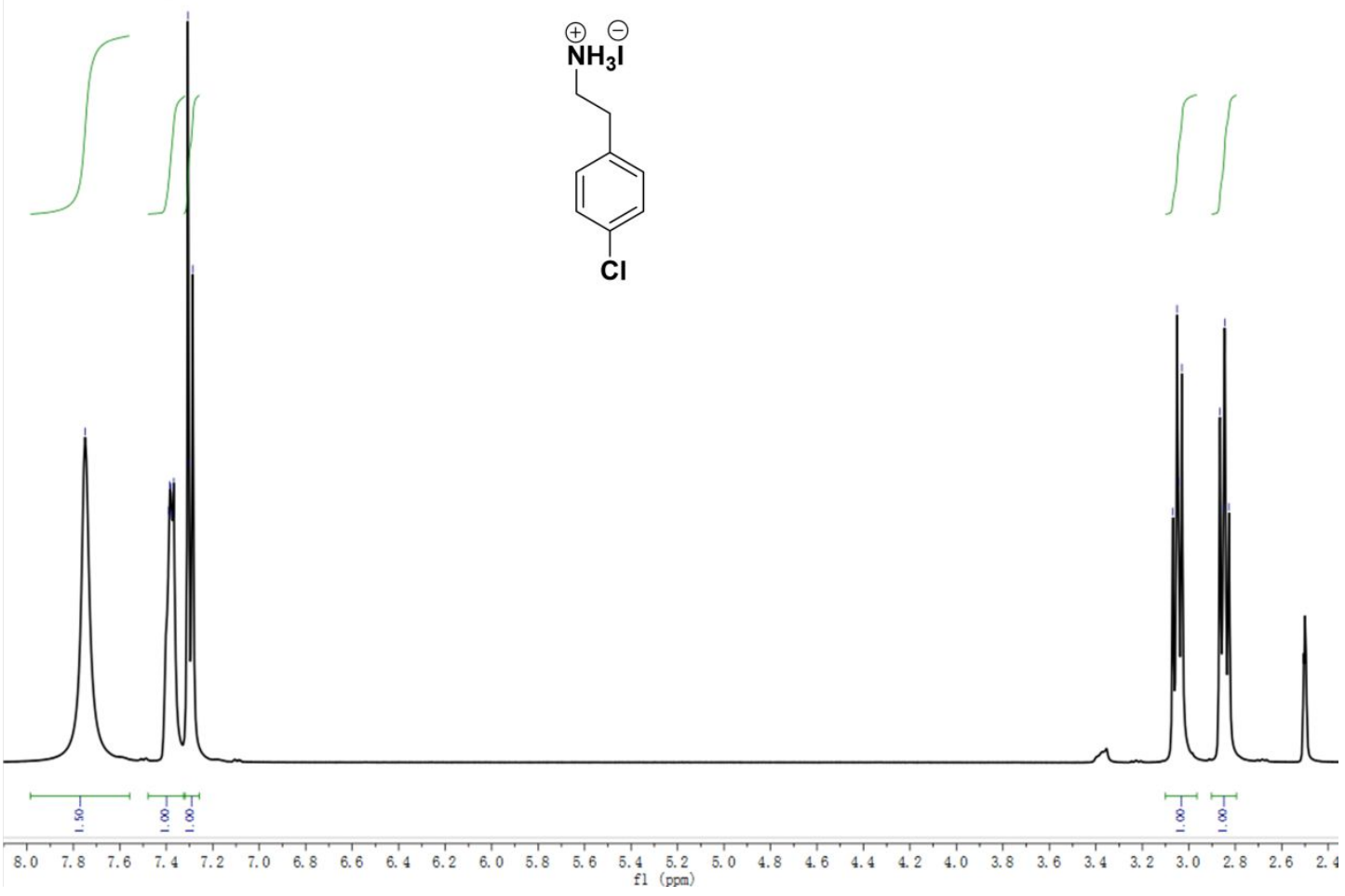


(d)

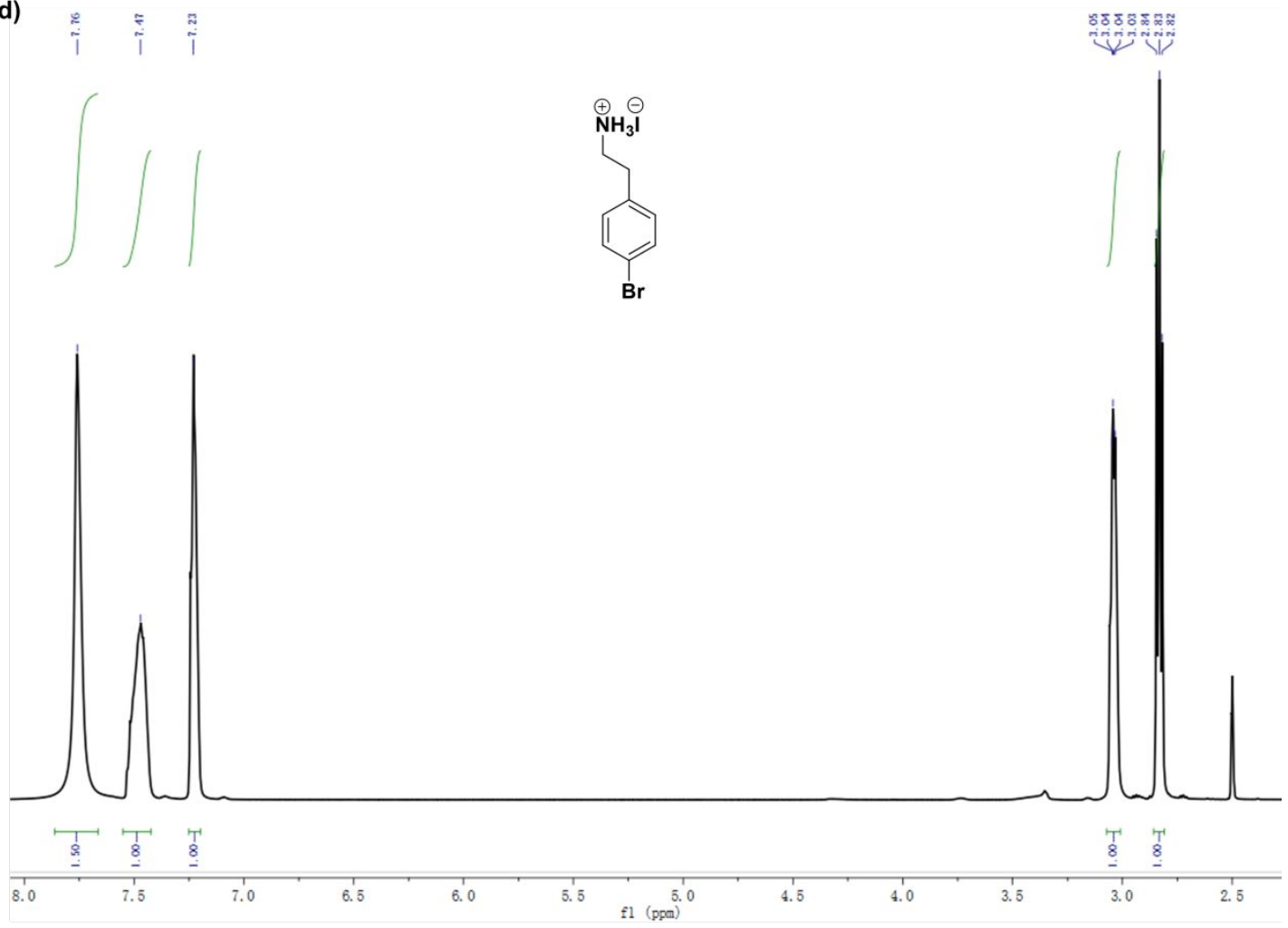

Figure S1. ${ }^{1} \mathrm{H}$ NMR of the (a) H-PEAI and X-PEAI, $\mathrm{X}=$ (b) $\mathrm{F}$, (c) $\mathrm{Cl}$, and (d) Br perovskite films, respectively. 
Table S1. Crystallographic data for (X-PEA $)_{2} \mathrm{PbI}_{4}(\mathrm{X}=\mathrm{F}, \mathrm{Cl}, \mathrm{Br})$.

\begin{tabular}{|c|c|c|c|}
\hline & $(\mathrm{F}-\mathrm{PEA})_{2} \mathrm{PbI}_{4}$ & $(\mathrm{Cl}-\mathrm{PEA})_{2} \mathrm{PbI}_{4}$ & $(\mathrm{Br}-\mathrm{PEA})_{2} \mathrm{PbI} 4$ \\
\hline $\begin{array}{l}\text { Empirical } \\
\text { formula }\end{array}$ & $\mathrm{C}_{16} \mathrm{H}_{22} \mathrm{~F}_{2} \mathrm{~N}_{2} \mathrm{PbI}_{4}$ & $\mathrm{C}_{16} \mathrm{H}_{22} \mathrm{C}_{12} \mathrm{~N}_{2} \mathrm{PbI}_{4}$ & $\mathrm{C}_{16} \mathrm{H}_{22} \mathrm{Br}_{2} \mathrm{~N}_{2} \mathrm{PbI}_{4}$ \\
\hline weight,g.mol ${ }^{-1}$ & 995.14 & 1028.04 & 1116.96 \\
\hline Temperature, $\mathrm{K}$ & $295(2)$ & $295(2)$ & $295(2)$ \\
\hline Wavelength, $\AA$ & 1.54184 & 1.54184 & 1.54178 \\
\hline Crystal system & Monoclinic & Monoclinic & Orthorhombic \\
\hline Space group & $\mathrm{P} 2 / \mathrm{c}$ & $\mathrm{C} 2 / \mathrm{c}$ & Cmca \\
\hline $\mathrm{a}, \AA$ & $16.71482(7)$ & $33.8513(11)$ & $8.59910(10)$ \\
\hline $\mathrm{b}, \AA$ & $8.62934(4)$ & $8.7102(3)$ & $8.79420(10)$ \\
\hline $\mathrm{c}, \AA$ & $8.80286(4)$ & $8.7716(3)$ & $35.3717(4)$ \\
\hline$\alpha,{ }^{\circ}$ & $90^{\circ}$ & 90 & 90 \\
\hline$\beta, \circ$ & $98.7424(4)^{\circ}$ & $93.647(3)$ & 90 \\
\hline$\gamma, \circ$ & $90^{\circ}$ & 90 & 90 \\
\hline Volume, $\AA^{3}$ & $1254.954(10)$ & $2581.08(15)$ & $2674.89(5)$ \\
\hline $\mathrm{Z}$ & 2 & 4 & 4 \\
\hline $\begin{array}{c}\text { Density } \\
\text { (calculated), } \\
\mathrm{mg} / \mathrm{m}^{3}\end{array}$ & 2.634 & 2.646 & 2.774 \\
\hline $\begin{array}{c}\text { Absorption } \\
\text { coefficient, } \mathrm{mm}^{-} \\
1\end{array}$ & 51.858 & 52.207 & 52.039 \\
\hline $\mathrm{F}(000)$ & 888 & 1840 & 1984 \\
\hline $\begin{array}{l}\text { Crystal size, } \\
\mathrm{mm}^{3}\end{array}$ & $\begin{array}{c}0.220 \times 0.120 \times \\
0.100\end{array}$ & $\begin{array}{c}0.240 \times 0.200 \times \\
0.200\end{array}$ & $\begin{array}{c}0.200 \times 0.160 \times \\
0.100\end{array}$ \\
\hline$\theta$ range, ${ }^{\circ}$ & 2.675 to 73.910 & 2.616 to 73.652 & 5.001 to $73.927^{\circ}$ \\
\hline Index ranges & $-20<=\mathrm{h}<=20$ & $-41<=\mathrm{h}<=42$ & $-10<=\mathrm{h}<=10$ \\
\hline
\end{tabular}




\begin{tabular}{|c|c|c|c|}
\hline & $\begin{array}{l}-10<=\mathrm{k}<=10, \\
-10<=\mathrm{l}<=10\end{array}$ & $\begin{array}{l}-10<=\mathrm{k}<=9 \\
-10<=1<=6\end{array}$ & $\begin{array}{l}-10<=\mathrm{k}<=10 \\
-43<=\mathrm{k}<=40\end{array}$ \\
\hline $\begin{array}{l}\text { Reflections } \\
\text { collected }\end{array}$ & 25213 & 13224 & 14505 \\
\hline Independent & $2534[\mathrm{R}($ int $)=$ & $2584[\mathrm{R}(\mathrm{int})=$ & $1443[\mathrm{R}(\mathrm{int})=$ \\
\hline reflections & $0.1054]$ & $0.0545]$ & $0.0439]$ \\
\hline $\begin{array}{c}\text { Completeness to } \\
\qquad \text { max }\end{array}$ & $100.0 \%$ & $100.0 \%$ & $99.9 \%$ \\
\hline $\begin{array}{l}\text { Max. and min. } \\
\text { transmission }\end{array}$ & $\begin{array}{l}1.00000 \\
0.06767\end{array}$ & $\begin{array}{l}1.00000 \\
0.20226\end{array}$ & $\begin{array}{l}1.00000, \\
0.16630\end{array}$ \\
\hline $\begin{array}{c}\text { Data / restraints / } \\
\text { parameters }\end{array}$ & 2534 / 0 / 117 & $2584 / 0 / 116$ & 1443 / 84 / 103 \\
\hline $\begin{array}{l}\text { Goodness-of-fit } \\
\text { on } \mathrm{F}^{2}\end{array}$ & 1.073 & 1.114 & 1.149 \\
\hline Final $\mathrm{R}$ indices & $\mathrm{R} 1=0.0437$ & $\mathrm{R} 1=0.0325$ & $\mathrm{R} 1=0.0237$ \\
\hline$[\mathrm{I}>2 \operatorname{sigma}(\mathrm{I})]$ & $\mathrm{wR} 2=0.1160$ & $\mathrm{wR} 2=0.0857$ & $\mathrm{wR} 2=0.0572$ \\
\hline $\begin{array}{l}\mathrm{R} \text { indices (all } \\
\text { data) }^{a}\end{array}$ & $\begin{array}{c}\mathrm{R} 1=0.0446, \\
\mathrm{wR} 2=0.1173\end{array}$ & $\begin{array}{c}\mathrm{R} 1=0.0343 \\
\mathrm{wR} 2=0.0870\end{array}$ & $\begin{array}{c}\mathrm{R} 1=0.0250 \\
\mathrm{wR} 2=0.0580\end{array}$ \\
\hline $\begin{array}{l}\text { Largest diff. } \\
\text { peak and hole, } \\
\text { e. } \AA^{-3}\end{array}$ & $3.781,-1.294$ & $1.799,-0.947$ & $0.937,-0.517$ \\
\hline
\end{tabular}

${ }^{a} R 1=\Sigma|| F_{\mathrm{O}}|-| F \mathrm{c} \| / \Sigma\left|F_{\mathrm{O}}\right|, \mathrm{wR}=\left\{\Sigma\left[\mathrm{w}\left(|\mathrm{Fo}|^{2}-|\mathrm{Fc}|^{2}\right)^{2}\right] / \Sigma\left[\mathrm{w}\left(|\mathrm{Fo}|^{4}\right)\right]\right\}^{1 / 2}$ 
(a)

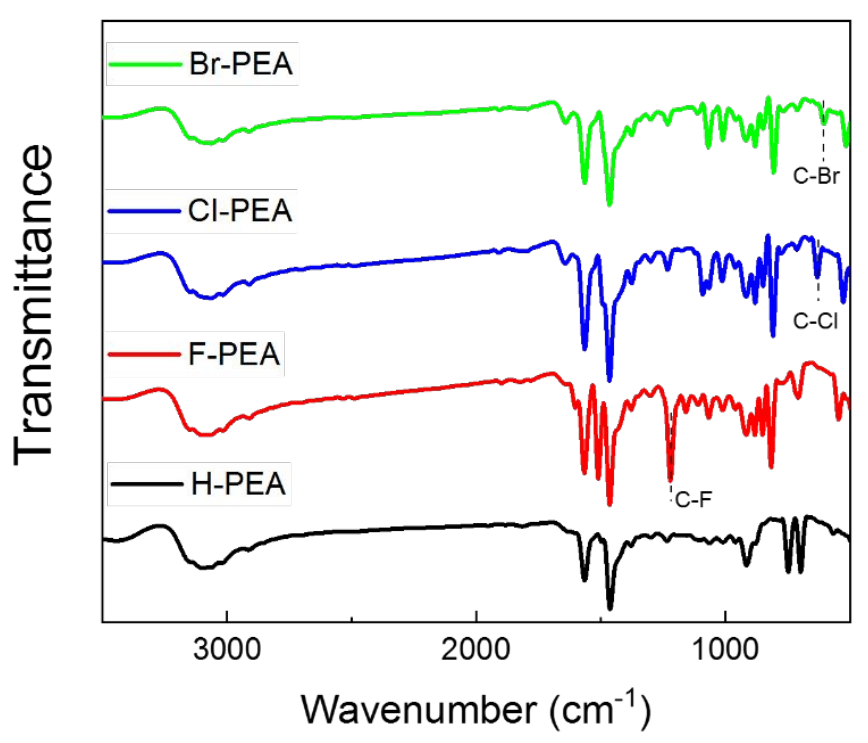

(b)

(c)
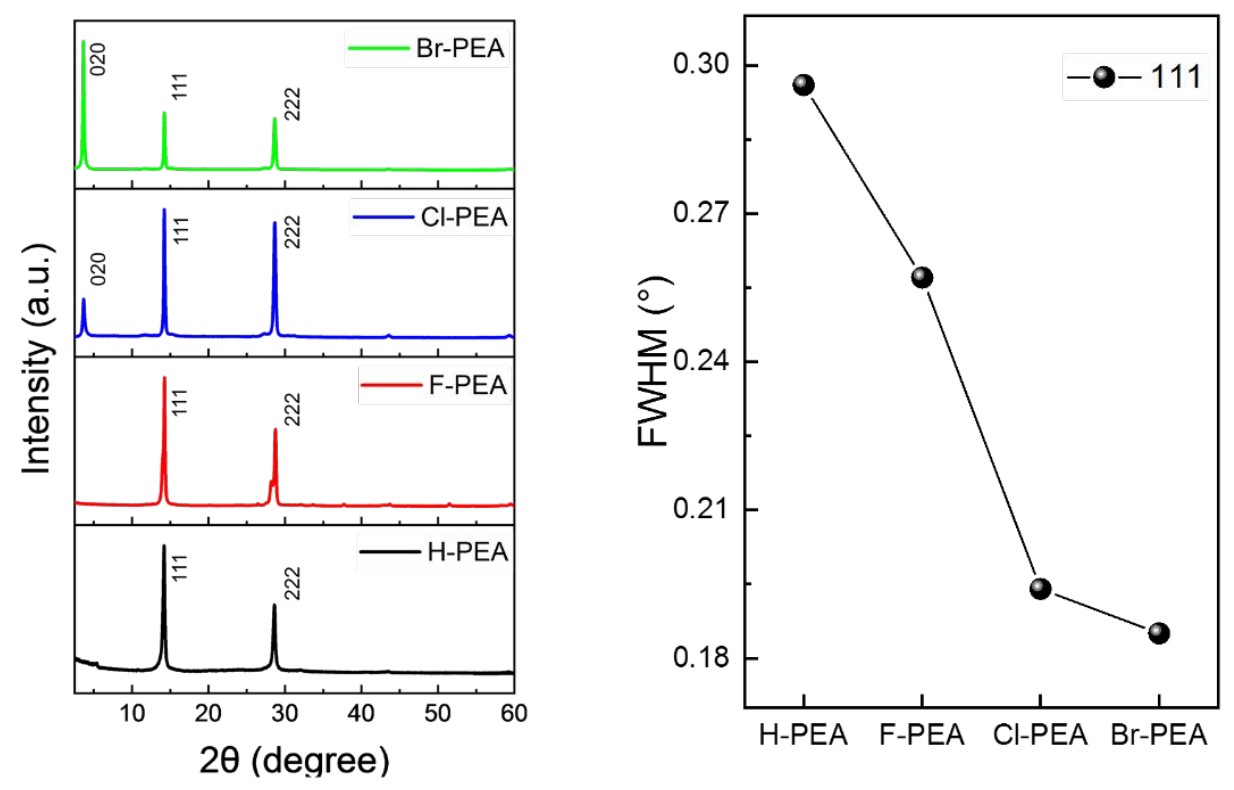

Figure S2. (a) FTIR spectra of the H-PEA and X-PEA (X=F, Cl, Br). (b)X-ray diffraction patterns of the H-PEA and X-PEA ( $\mathrm{X}=\mathrm{F}, \mathrm{Cl}, \mathrm{Br})$. (c)FWHM of the H-PEA and X-PEA (X=F, Cl, Br) for the diffraction peaks of the (111) plane. 
(a)

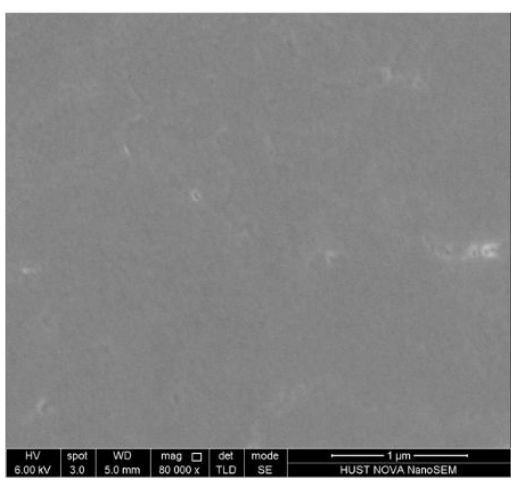

(c)

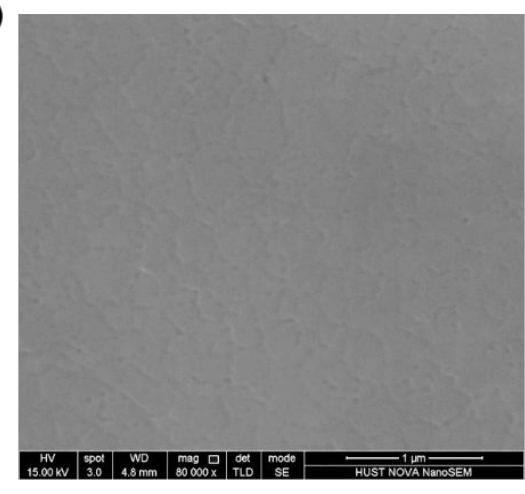

(b)

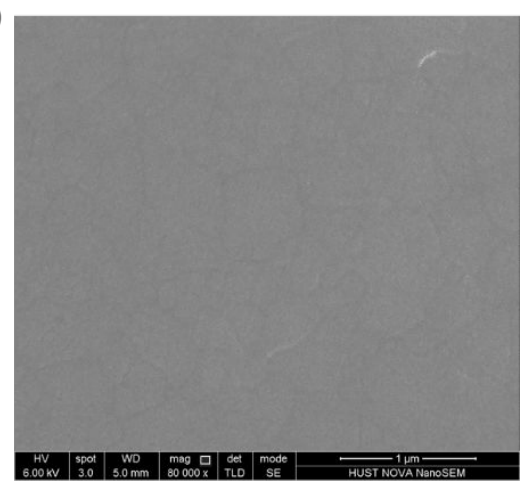

(d)

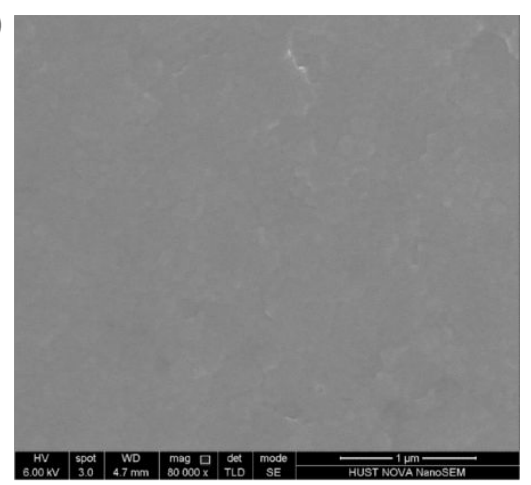

Figure S3. SEM images of the (a) H-PEA, and X-PEA, $\mathrm{X}=$ (b) F, (c) Cl, and (d) Br perovskite films, respectively. 


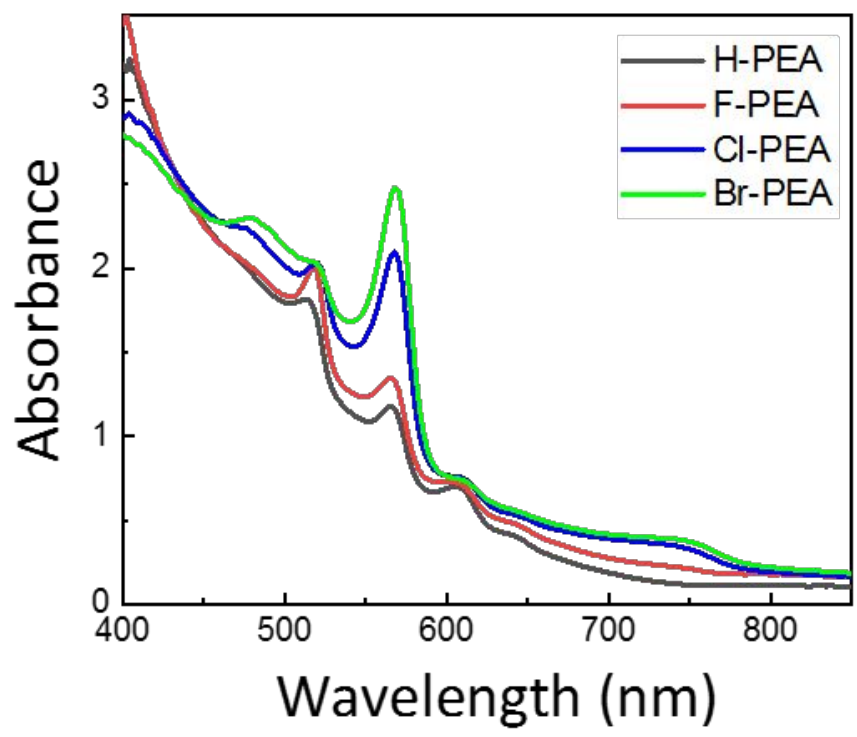

Figure S4. Absorption of the H-PEA and X-PEA (X=F, Cl, Br) perovskite films, respectively.
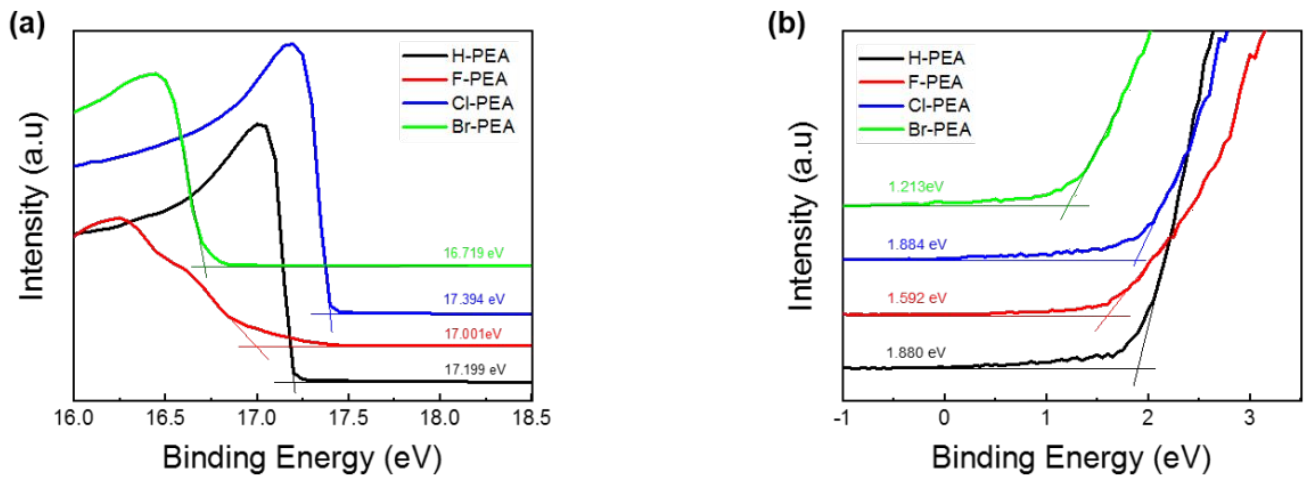

Figure S5. UPS spectra describing the (a) cutoff energy $\left(\mathrm{E}_{\text {cutoff }}\right)$ and (b) Feimi edge ( $\mathrm{E}_{\mathrm{F}}$, edge) of H-PEA and X-PEA (X=F, Cl, Br) perovskite films. 
Table S3. Fitting results of PL decay lifetime of H-PEA and X-PEA $(\mathrm{X}=\mathrm{F}, \mathrm{Cl}, \mathrm{Br})$ films.

\begin{tabular}{ccccc}
\hline Film & $\tau_{1}(\mathrm{~ns})$ & $\begin{array}{c}\text { weight } \\
\text { fraction }\end{array}$ & $\tau_{2}(\mathrm{~ns})$ & $\begin{array}{c}\text { weight } \\
\text { fraction }\end{array}$ \\
\hline H-PEA & 4.25 & $95.24 \%$ & 31.60 & $4.76 \%$ \\
F-PEA & 10.85 & $87.95 \%$ & 72.00 & $12.05 \%$ \\
Cl-PEA & 11.98 & $84.72 \%$ & 50.12 & $15.28 \%$ \\
Br-PEA & 9.77 & $77.99 \%$ & 43.58 & $22.01 \%$ \\
\hline
\end{tabular}



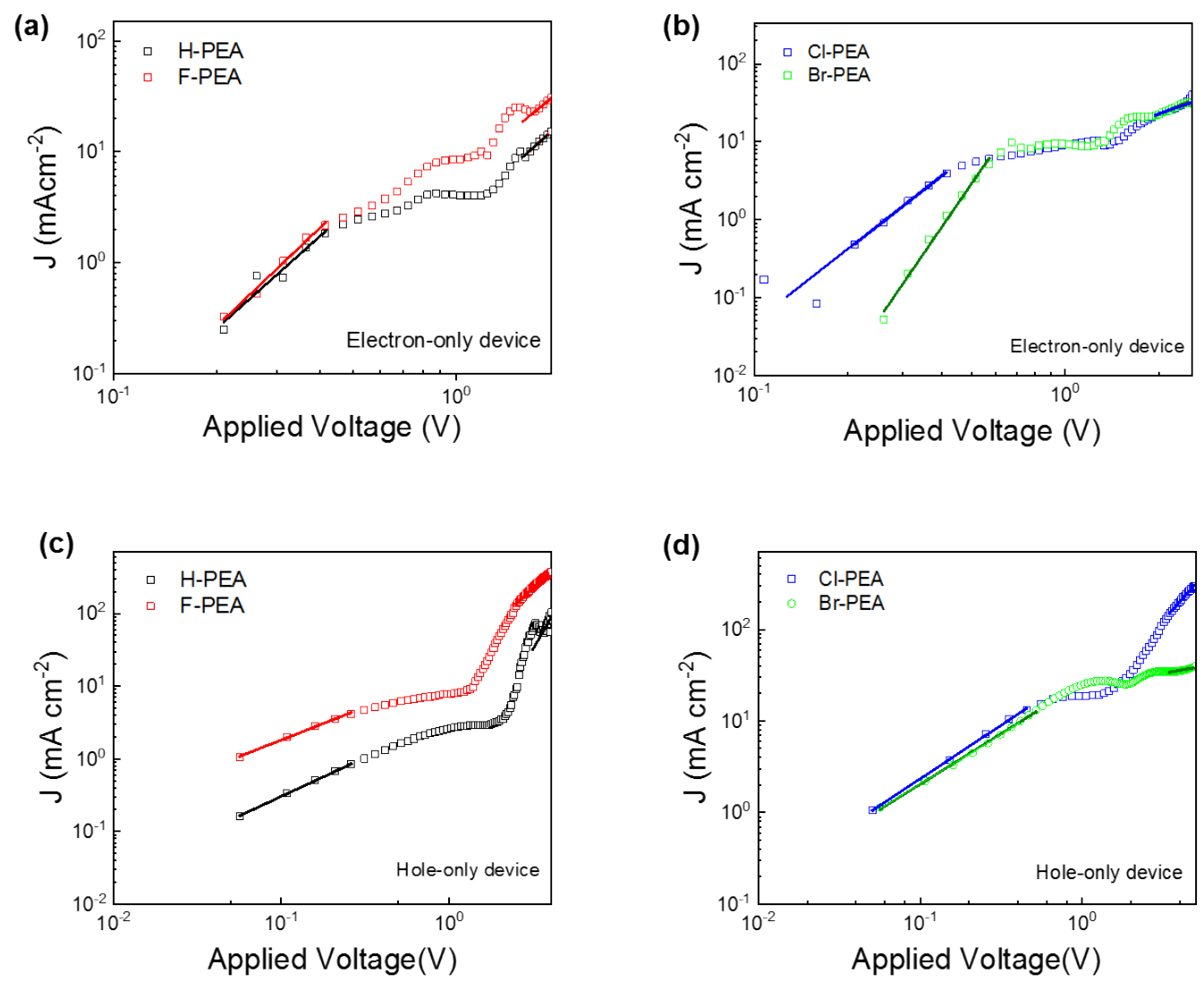

Figure S6. Dark J-V measurements of electron-only devices for (a) H-PEA and F-PEA and (b) ClPEA and Br-PEA perovskite and of hole-only devices for (c) H-PEA and F-PEA and (d) Cl-PEA and Br-PEA perovskite films. 


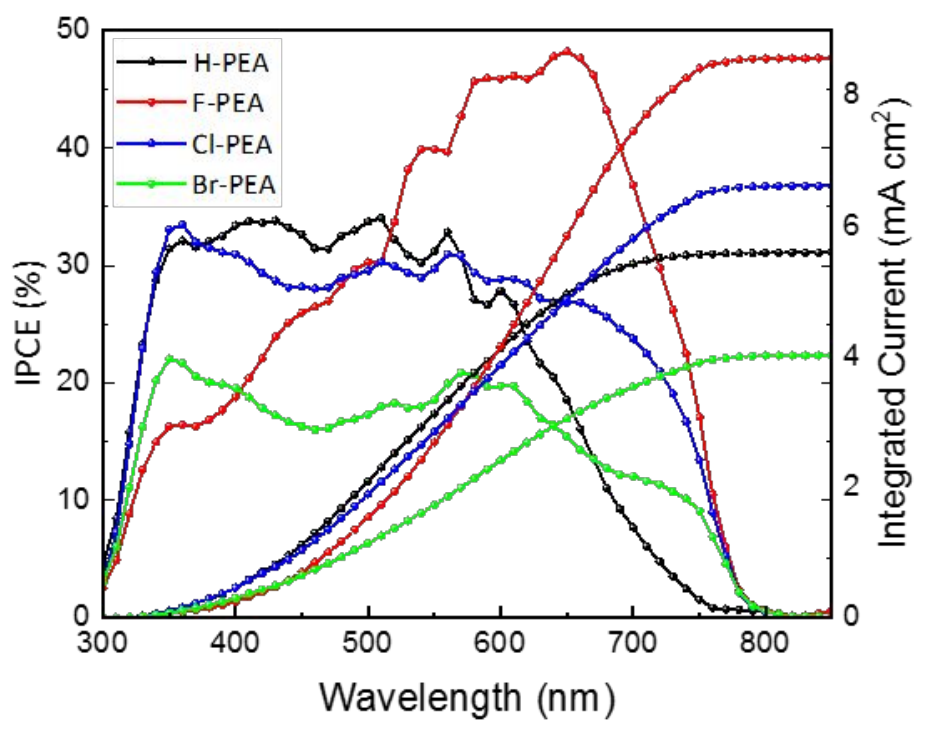

Figure S7. IPCE spectra and the integrated current of the devices based on H-PEA and X-PEA $(\mathrm{X}=\mathrm{F}, \mathrm{Cl}, \mathrm{Br})$ perovskite solar cells. 